\title{
Electronic and optical properties of the group IV doped copper gallium chalcopyrites
}

\author{
C. Tablero \\ Instituto de Energía Solar, Universidad Politécnica de Madrid, Ciudad Universitaria s/n, 28040 Madrid, Spain
}

\section{A R T I C L E I N F O}

\section{Keywords:}

Electronic structure

Chalcopyrite

Impurities

Optical properties

\begin{abstract}
A B S T R A C T
Ternary chalcopyrite semiconductors are well known as promising materials for photovoltaic applications. The substitution of group IV atoms in sulfur and selenium copper gallium chalcopyrites $\mathrm{GaCuX}_{2}$, with $\mathrm{X}=\mathrm{S}$ and Se, could have important implications either for photovoltaic or spintronic applications. To better understand these effects, we have made first-principles calculations on the doped chalcopyrites with group IV atoms, with different concentrations and geometries. Many of these materials have an intermediate band that modifies the optical properties of the host semiconductor. The sub-gap absorptions have also been determined using the absorption coefficients.
\end{abstract}

\section{Introduction}

The photovoltaic market is currently dominated by silicon technologies. However, the challenge is to manufacture more cost effective solar cell materials and maintain a high efficiency. Chalcopyrites are one of the most promising options because for their use as light absorbing materials in thin-film solar cells [1-5]. These types of materials have increased in importance as solar absorbers in thin-film solar cells, because they enable the most efficient thin-film devices to be produced. For this reason alloys of chalcopyrite semiconductors have recently become of interest for their role in improving the efficiencies of sulfur and selenium chalcopyrite solar cells [6-10]. However, the effect of alloying on the defects in these materials is still largely unexplored. The opportunity of $\mathrm{M}$ doping in $\mathrm{GaCuX}_{2}$ chalcopyrite has been discussed in Ref. [19] in accordance with thermo-chemical analyses. Elements of the group VIIIb and IVa have been identified as interesting candidates to act as substitutes in the cation sublattice of the chalcopyrite hosts.

The occurrence of a metallic band in the gap at high impurity concentration can also increase the efficiency with respect to the host semiconductor [11,12]. Solar cell devices with a partially full intermediate band (IB) have a theoretical limiting efficiency of $63.2 \%$ [11,12], in contrast to $40.7 \%$ for conventional single-gap solar cells [13]. These materials have been studied in some detail in recent years [14-17].

Both sulfur and selenium chalcopyrites, belonging to the $\mathrm{GaCuX}_{2}$ and $\mathrm{InCuX}_{2}(\mathrm{X}=\mathrm{S}$ and $\mathrm{Se}$ ) family of compounds used in high efficiency chalcopyrite-based solar cells, have gaps close to the optimum for a thin-film IB solar cell. These materials have theoretical

E-mail address: ctablero@etsit.upm.es. radiative limiting efficiencies under one sun of more than $46 \%$ for $\mathrm{GaCuS}_{2}$ and more than $42 \%$ for $\mathrm{GaCuSe}_{2}$ [18].

The group IV atoms are promising candidates for doped thin-film IB solar cells [19]. It is thus interesting to see if the presence of these impurities is detrimental for the photovoltaic properties or whether they can be beneficial. In particular, in this work an analysis of the electronic and optical properties of the chalcopyrite structure $M_{x} A_{1-{ }_{x}} B X_{2}$ for different concentrations will be carried out, where $M$ is a group IV atom $(\mathrm{M}=\mathrm{C}, \mathrm{Si}, \mathrm{Ge}$, and $\mathrm{Sn})$ and $\mathrm{X}=\mathrm{S}$ and $\mathrm{Se}$. The two possible substitutions, $\mathrm{M}$ by $\mathrm{Ga}(\mathrm{A}=\mathrm{Ga}$ and $\mathrm{B}=\mathrm{Cu})$ or $\mathrm{M}$ by $\mathrm{Cu}(\mathrm{A}=\mathrm{Cu}$ and $\mathrm{B}=\mathrm{Ga})$ are

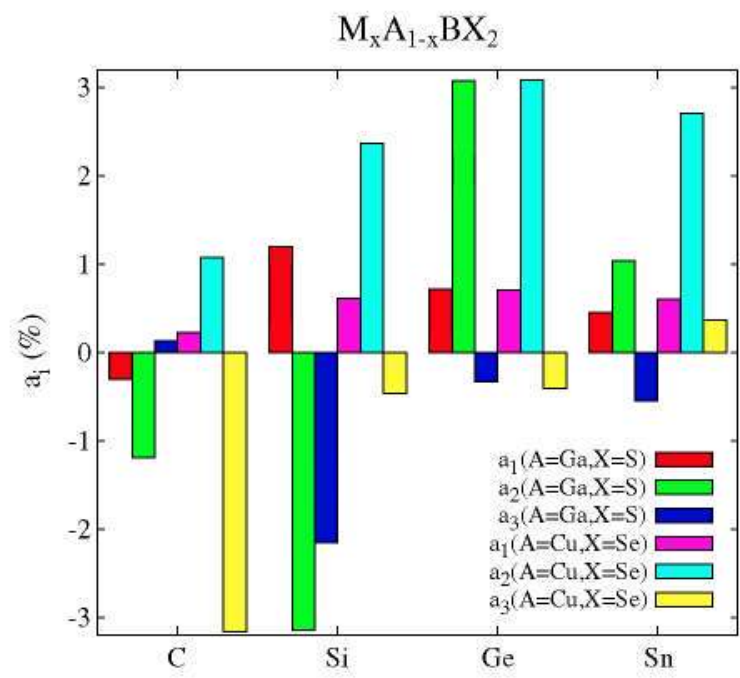

Fig. 1. Relative deviation (\%) of the lattice vector modules $\left(a_{i,} i=1,2,3\right)$ of the substituted chalcopyrite $\left(M_{x} A_{1-x} B X_{2}\right)$ with respect to the chalcopyrite without substitution. 
analyzed. Firstly the electronic spectrum is obtained in order to verify whether these alloys have the required IB in the chalcopyrite host. Finally the absorption coefficients are obtained in order to analyze the sub-gap transitions.

\section{Calculations}

In order to obtain the electronic properties we use the densityfunctional theory (DFT) [20-24] in the local-spin density approximation (LSDA) with periodic boundary conditions. The defect calculations are made by replacing one or several $\mathrm{Cu}$ or $\mathrm{Ga}$ atoms with other atoms in the chalcopyrite structure $\left(M_{x} A_{1-}{ }_{x} B X_{2}\right.$ with $(A, B)=(G a$, $\mathrm{Cu}), \mathrm{X}=\mathrm{S}$ and $\mathrm{Se}$, and $\mathrm{M}=\mathrm{C}, \mathrm{Si}, \mathrm{Ge}$, and $\mathrm{Sn}$ ). The concentrations analyzed are from $\mathrm{x}=1 / 4$ to $\mathrm{x}=1 / 54$. The standard Kohn-Sham (KS)
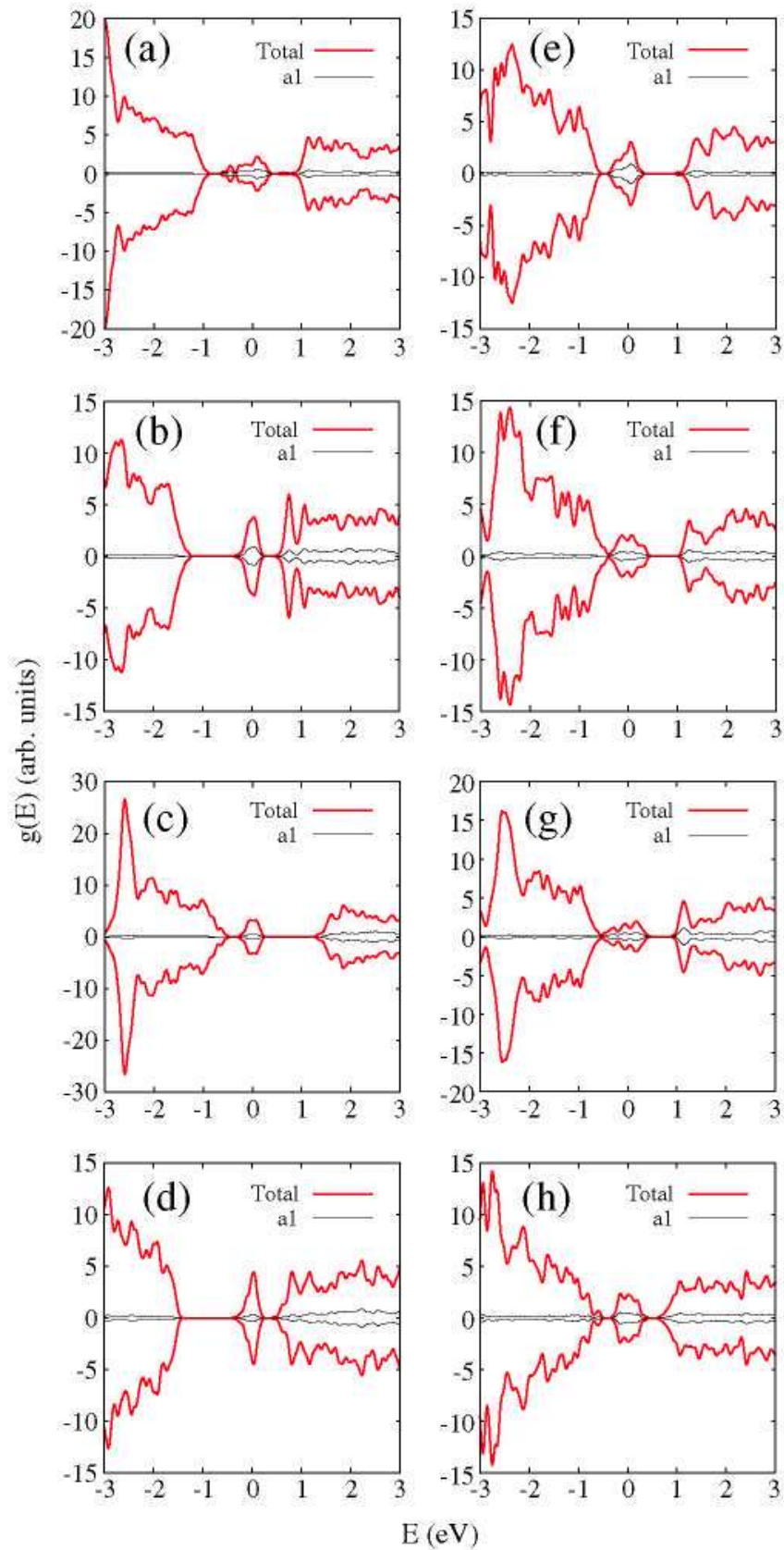

Fig. 2. Total density of states (thick line, indicated by "Total" in the panels) and projected density of states on the impurity atom $\mathrm{M}$ (the thin line, indicated by " $a 1$ " in the panels) for the chalcopyrite $\mathrm{M}_{x} \mathrm{Ga}_{1-x} \mathrm{CuS}_{2}$ with $\mathrm{M}=\mathrm{C}$ (a), Si (b), Ge (c) and $\mathrm{Sn}$ (d), and $x=1 / 4$. Similar for (e), (f), (g) and (h), but for the chalcopyrite $\mathrm{M}_{x} \mathrm{Cu}_{1-x} \mathrm{GaS}_{2}$. The Fermi energy as zero has been chosen in this figure.
[25] equations are solved selfconsistently [26]. For the exchange and correlation term, the LSDA has been used as proposed by Ceperley and Alder [27]. The standard Troullier-Martins [28] pseudopotential is adopted and expressed in the Kleinman-Bylander $[29,30]$ factorization. The KS orbitals are represented using a linear combination of confined pseudoatomic orbitals [31].

In all calculations, from a double-zeta with polarization (DZP) to a triple-zeta with polarization (TZP) basis set for all atoms have been used. The results using the bases DZP and TZP basis set are similar. The results that we will present correspond to the TZP basis set on all the atoms.

We have made calculations for three different nuclear configurations: (i) the atoms in the same positions as the experimental chalcopyrite, (ii) the position after optimizing the internal atomic positions with the size and symmetry of the experimental chalcopyrite cell (i.e. with symmetry constraints), and (iii) the positions after optimizing both, the internal cell positions and the lattice vectors (i.e.,
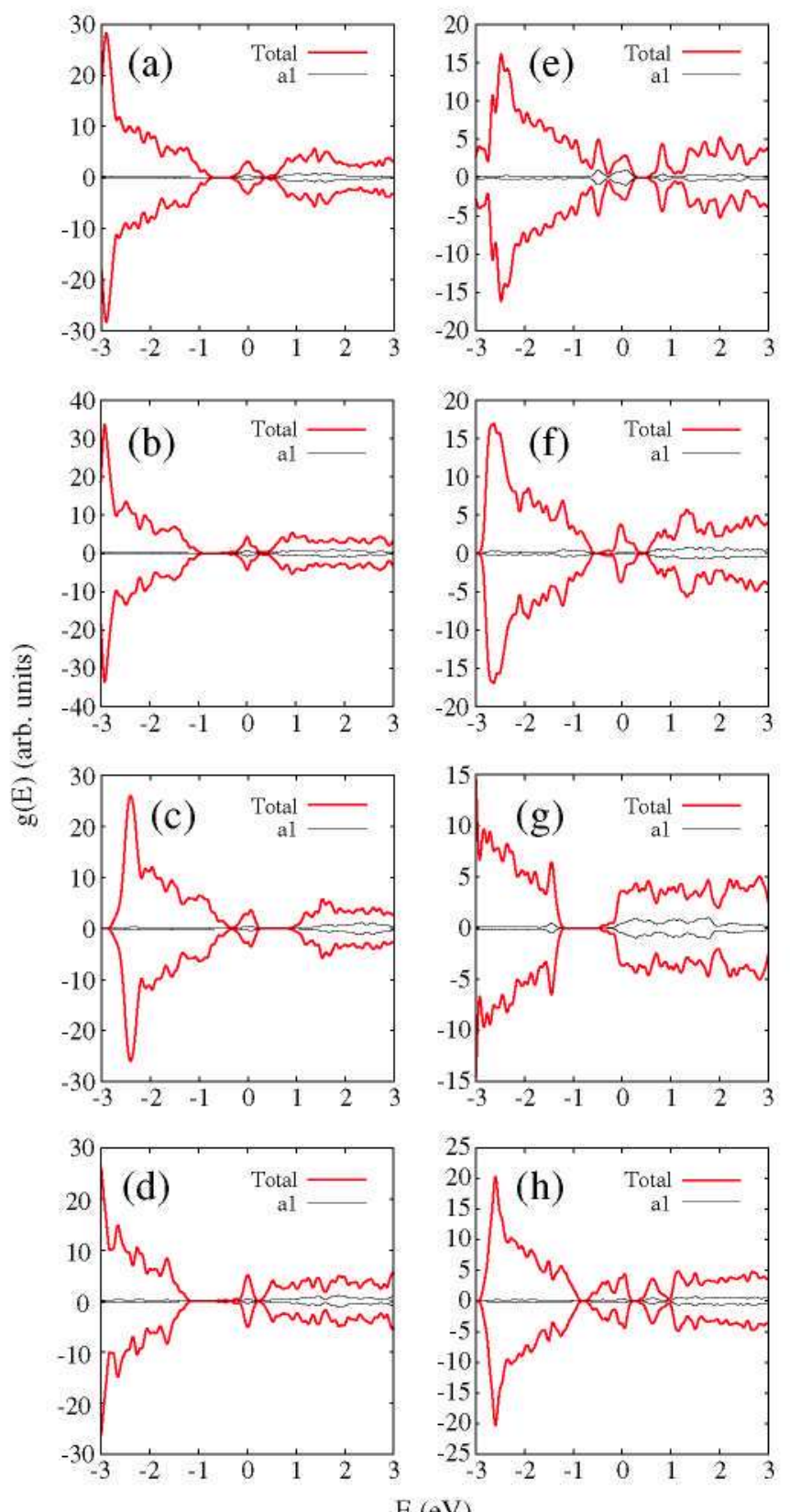

$\mathrm{E}(\mathrm{eV})$

Fig. 3. Same legend as in Fig. 2, but for the selenium chalcopyrite. $M_{x} A_{1-x} B S e_{2}$. 
without symmetry constraints). In the latter cases, the atom positions were allowed to relax in accordance with the calculated quantum mechanical forces until the total energy minimum was reached and the forces became less than $0.04 \mathrm{eV} / \AA$.

To determine the optical properties, the complex dielectric function is calculated as a function of the photon energy ( $\hbar \omega)$, the energy $\left(E_{\mu, \vec{k}}\right)$ and occupations $\left(f_{\mu \vec{k}}\right)$ of the $\mu$ band at point $\vec{k}$ of the Brillouin zone (BZ), and the momentum matrix elements:

$\varepsilon_{2}(\omega) \sim \frac{1}{\omega^{2}} \sum_{\vec{k}} \sum_{v} \sum_{c}|<v, \vec{k}| \hat{p}|c, \vec{k}>|^{2}\left[f_{v, \vec{k}}-f_{c, \vec{k}}\right] \times \delta\left(E_{c, \vec{k}}-E_{v, \vec{k}}-\hbar \omega\right)$

The other optical properties are obtained using the Kramers-Kroning relations. In order to obtain $\varepsilon_{2}$, the occupation factors $f_{\mu, \vec{k}}$ and the energies $E_{\mu, \vec{k}}$ are determined from the aforementioned total energy calculation. The momentum operator optical matrix elements $<\mathrm{v}, \vec{k}|\hat{\mathrm{p}}| c, \vec{k}>$ between the states $\mid v, \vec{k}>$ and $\mid c, \vec{k}>$ of the $v$ and the $c$ bands at point $\vec{k}$ of the $\mathrm{BZ}$ are calculated directly. To consider the absorption process overall, we need to include the occupation factors to represent the absorption process from the $v$ to the $c$ bands $\left(f_{v, \vec{k}}\left[1-f_{c, \vec{k}}\right]\right)$ and the emission process from the $c$ to the $v$ bands $\left(f_{c, \vec{k}}\left[1-f_{v, \vec{k}}\right]\right)$. Then, the occupation factors for the overall absorption + emission process are $\left[f_{c, k}-f_{v, \vec{k}}\right]$.

\section{Results and discussion}

The results for the host chalcopyrites $\mathrm{GaCuX}_{2}$ are compared well with the results in the literature. For example, the gaps obtained with the three aforementioned nuclear configurations in the calculation section are $0.86 \mathrm{eV}, 1.02 \mathrm{eV}$ and $1.13 \mathrm{eV}$ for $\mathrm{X}=\mathrm{S}$, and $0.84 \mathrm{eV}, 0.70 \mathrm{eV}$ y $0.70 \mathrm{eV}$ for $\mathrm{X}=\mathrm{Se}$. Other results in the literature are $1.25 \mathrm{eV}$ [32], $0.69 \mathrm{eV}$ [33] and $0.92 \mathrm{eV} \mathrm{[34]} \mathrm{for} \mathrm{X=S,} \mathrm{and} 0.48 \mathrm{eV}$ [32] and $0.03 \mathrm{eV}$ [33] for $X=S e$, whereas the experimental results are $2.43 \mathrm{eV}$ and $1.68 \mathrm{eV}$ for $\mathrm{X}=\mathrm{S}$ and Se respectively. Because of the use of the DFTLSDA we have the well known gap subestimation problem [19]. The

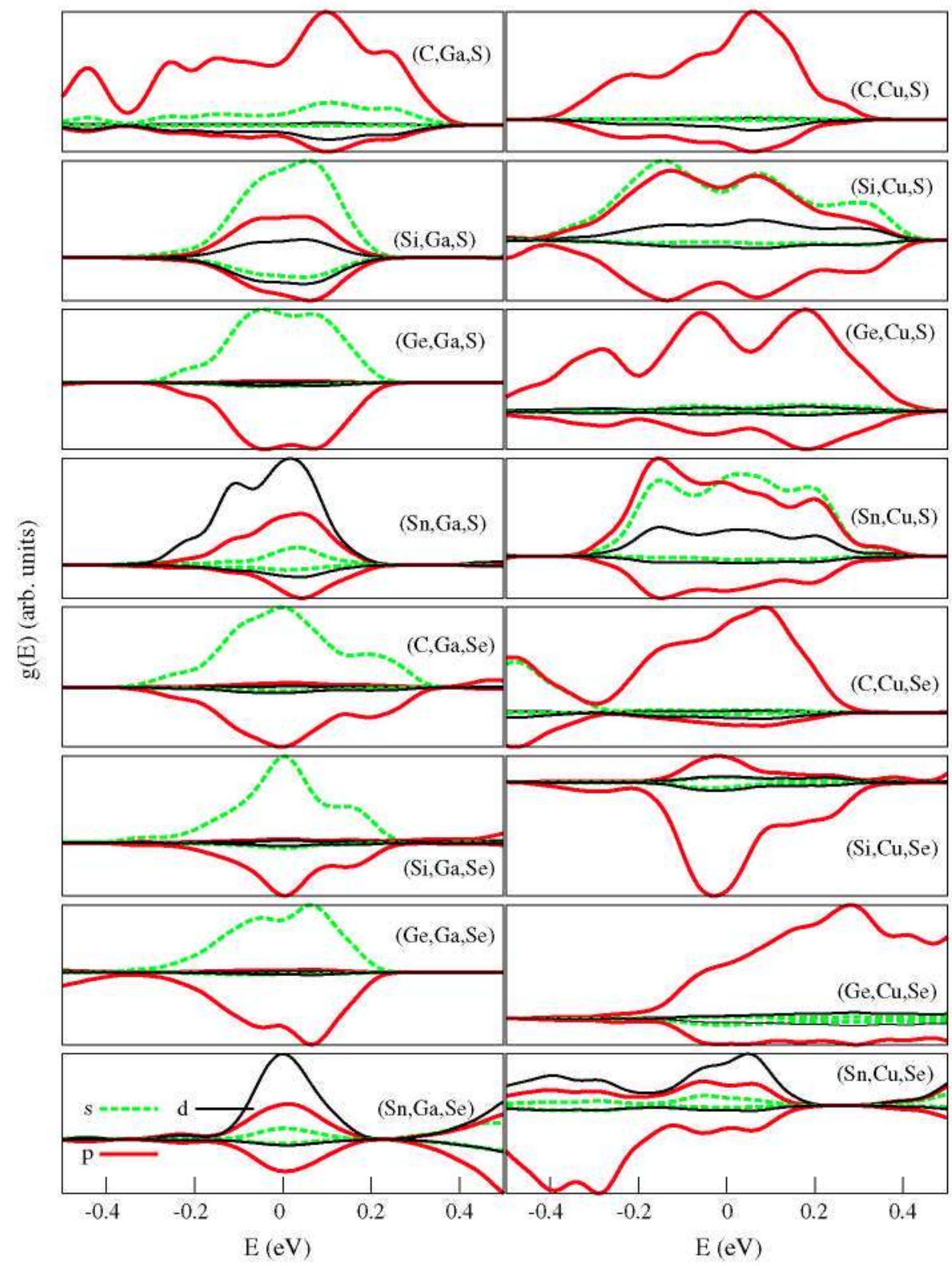

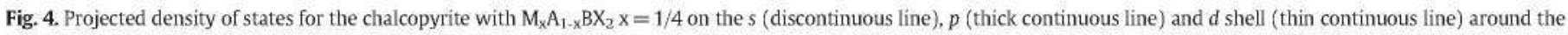

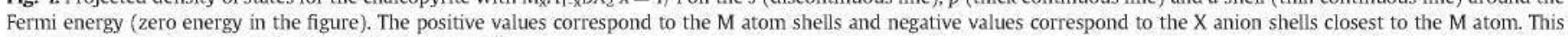
chalcopyrite is indicated by (M, A and X) in the panel title. 
distances from cation to the four anion's nearest neighbors after optimization of the atomic positions are $2.30 \AA$ and $2.43 \AA$ for $\mathrm{X}=\mathrm{S}$ and Se respectively. The experimental distances are $2.34 \AA$ and $2.42 \AA$. Therefore the relaxation of all the atoms leads to a decrease in these distances in the case of $\mathrm{X}=\mathrm{S}$, while they are almost the same for $\mathrm{X}=$ Se.

For the substituted chalcopyrite $M_{x} A_{1-x} B X_{2}$, the lattice parameters and the initial tetrahedral distribution of the first neighbors $\mathrm{X}$ around $\mathrm{M}$ is distorted. The experimental lattice vector modules are $\mathrm{a}_{1}=\mathrm{a}_{2}=5.35 \AA$, and $\mathrm{a}_{3}=6.45 \AA$ for the sulfur host chalcopyrite, and $a_{1}=a_{2}=5.61 \AA$, and $a_{3}=6.79 \AA$ for the host selenium chalcopyrite [32]. In Fig. 1, the deviation of the relaxed lattice vectors of the doped chalcopyrites with respect to the experimental lattice vectors of the host chalcopyrites is shown. The more distorted vector module corresponds to $a_{2}$ in almost all cases.

The electronic structure of these doped chalcopyrites is characterized in almost all cases, except when $\mathrm{Cu}$ is substituted by $\mathrm{Ge}$, by a partially full intermediate band (IB) in the gap. The structure of this band and the relative composition of the impurity atom can be seen in Figs. 2 and 3, where the total density of states and the density of states projected in the impurity atom are represented. The Fermi energy (zero energy in the figures) crosses the IB, indicating that this band is partially full.

Because of the DFT-LSDA gap subestimation, the absolute position of this band with respect to the valence band (VB) and to the conduction band $(\mathrm{CB})$ edges is difficult to determine. For the chalcopyrites, both the $\mathrm{VB}$ and the $\mathrm{CB}$ edges have a high contribution of the anion orbitals. The IB also has a contribution of the $\mathrm{X}$ anion orbitals. The two subgaps between the VB and the IB, and between the IB and the $\mathrm{CB}$ will be substimated similarly because their edges have the same anion character. Therefore it is expected that the subgaps increase with respect to that shown in Figs. 2 and 3. More accurate first-principles approaches [20-23] that avoid the band-gap problem, such as the Green function with screened interaction approximation, the hybrid functionals, the self-interaction-corrected density-functional theory, the exact exchange method, etc., are currently not practical in large supercells.

In order to analyze the orbital contribution to the IB of the $\mathrm{M}$ atoms and of the orbitals of the nearest anions, we show the projected density of states on the orbitals of symmetry $s, p$ and $d$ of $\mathrm{M}$ (positive region above zero in the Figure), and of the nearest anions to $M$ (negative region) in Fig. 4. In all of the cases, the IB is made up of a combination of the $\mathrm{M}$ states with the $\mathrm{sp}-\mathrm{X}$ states of the nearest anions. The $\mathrm{M}$ states that contribute more to the IB are mainly the $p$-M states in most cases. For $\mathrm{M}=\mathrm{Sn}$, the $d$-M states contribute even more than the $p-\mathrm{M}$ states. This is because, in this case, the orbital $d-\mathrm{M}$ is nearer in energy to the $p$-X orbital of the anion. Therefore its combination to form the states of the IB is more favorable. For the $\mathrm{M}_{\mathrm{X}} \mathrm{Ga}_{1-\mathrm{x}} \mathrm{CuS}_{2}$ with $\mathrm{M}=\mathrm{Si}$ and $\mathrm{Ge}$, the $s-\mathrm{M}$ symmetry state of $\mathrm{M}$ has the larger contribution to the IB. This same happens for the chalcopyrites $\mathrm{M}_{\mathrm{x}} \mathrm{Ga}_{1-\mathrm{x}} \mathrm{CuSe}_{2}$ with $\mathrm{M}=\mathrm{C}$, Si and $\mathrm{Ge}$.

The IB is present in all of the nuclear configurations analyzed: with the experimental positions, with the experimental lattice parameters and the internal atomic positions optimized, and with all position and lattice parameters optimized (see Figs. 2 and 3). This IB band is also present when we diminish the concentration. For example, the band diagrams for different values of $x$ are shown in Fig. 5 for the $\mathrm{Ge}_{\mathrm{x}} \mathrm{Ga}_{1-\mathrm{x}} \mathrm{CuS}_{2}$ chalcopyrite. For the lowest $\mathrm{M}=\mathrm{Ge}$ concentration (larger cells) we have used the experimental atomic positions of the chalcopyrite without substituting. Comparing Fig. 5 with Fig. $4 c$, the effects on the relaxation of the atomic positions are a small increase in the gap, a slight narrowing of the band in the gap and a displacement in energy of the band in the gap toward the VB.

The chalcopyrites are of the most absorbent materials of the solar spectrum. The edge of the VB is made up by combining the $d-\mathrm{Cu}$ orbitals with the $s p-\mathrm{X}$ anion orbitals. The edge of the $\mathrm{CB}$ also has a high
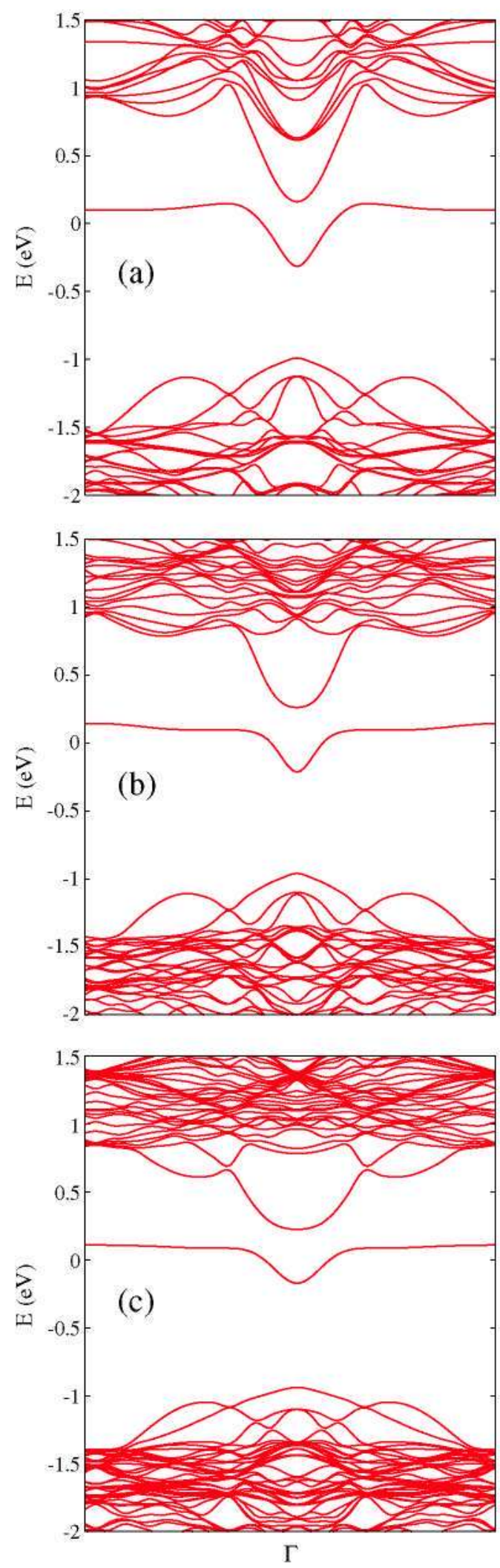

Fig. 5. Electronic structure around $\Gamma$ the point for $\mathrm{Ge}_{x} \mathrm{Ga}_{1-\mathrm{x}} \mathrm{CuS}_{2}$ with (a) $\mathrm{x}=1 / 16$, (b) $x=1 / 24 y$, and (c) $x=1 / 54$ using the atomic experimental positions. The Fermi energy as zero has been chosen in this figure.

contribution of $s p$-X anion orbitals. Because of the similar character of the $\mathrm{VB}$ and $\mathrm{CB}$ edges, the absorption has an intraatomic component and could be larger than for the binary compounds, where the VB and 


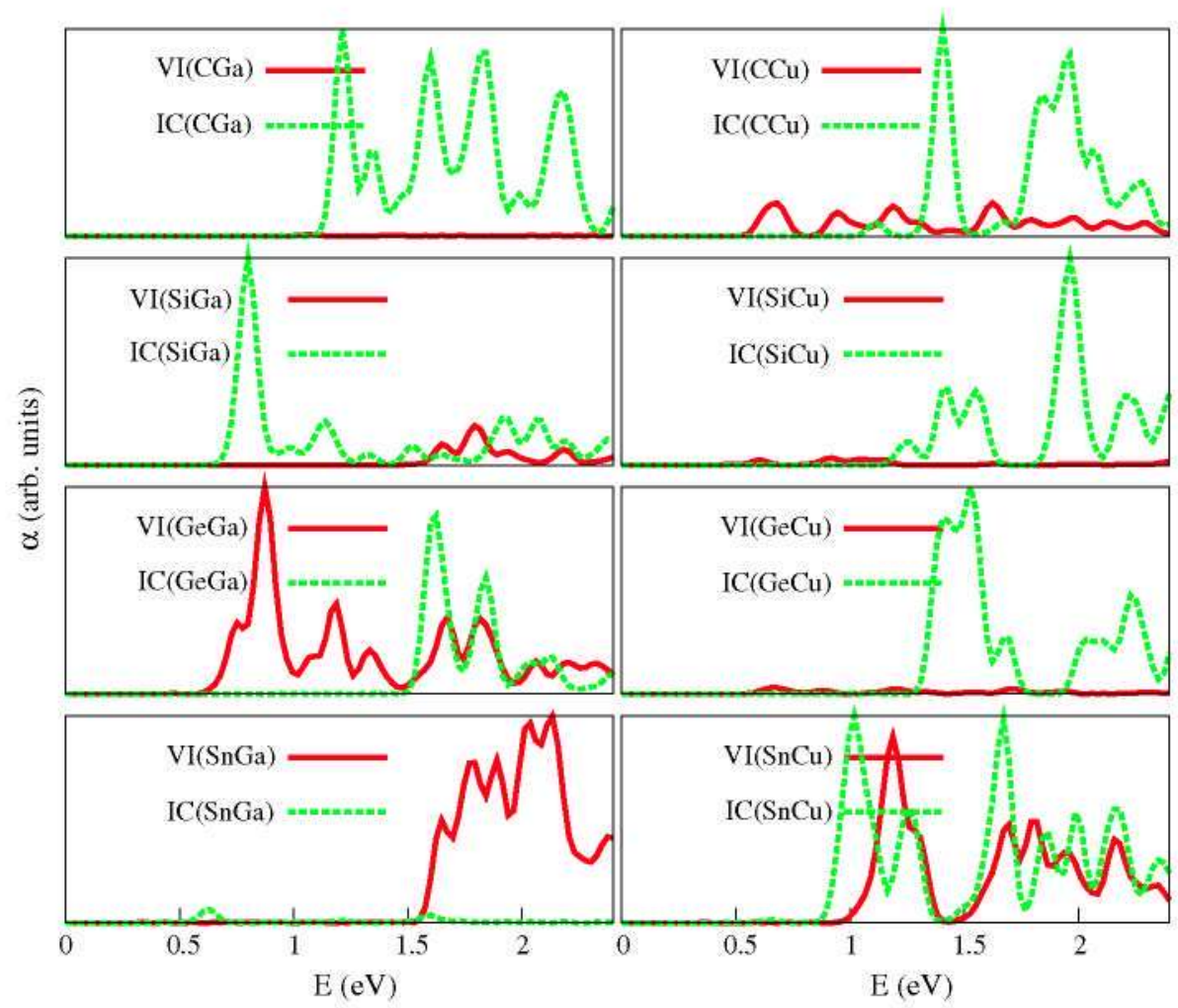

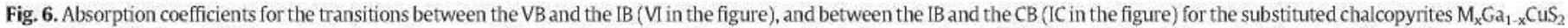
and $\mathrm{M}_{\mathrm{x}} \mathrm{Cu}_{1-\mathrm{x}} \mathrm{GaS}_{2}(\mathrm{x}=1 / 4)$. The substitution of the group IVa atom by the $\mathrm{Ga}$ or $\mathrm{Cu}$ atom is indicated in parenthesis: $(\mathrm{MGa})$ for $\mathrm{M}_{x} \mathrm{Ga}_{1-\mathrm{x}} \mathrm{CuS}$, and (MCu) for $\mathrm{M}_{x} \mathrm{Cu}_{1-\mathrm{x}} \mathrm{GaS}_{2}$.

the $\mathrm{CB}$ edges have an anionic and a cationic (interatomic) character respectively. It is due to a stronger overlap of the initial with the final wave functions for the intraatomic transitions.

Because the IB is partially full there are two possible additional transitions of lower energy photons, in addition to the usual transition from the VB to the $\mathrm{CB}$ : from the VB to the IB and from the IB to the $C B$. The usual transition from the $V B$ to the $C B$ generates an electron-hole. The transitions from the VB to the IB and from the IB to CB generate one hole and one electron additional respectively. Therefore the number of electrons in the $\mathrm{CB}$ and holes in the VB will be larger than for the host semiconductor. If these materials are used to absorb photons in a photovoltaic device instead of the host semiconductor, the current will be larger because of the two-transition sub-gap.

Finally, the contributions to the absorption from transitions with lower energy than the chalcopyrite host semiconductor gap are shown in Fig. 6. These transitions are from the VB to the IB and from the IB to the $\mathrm{CB}$. In most cases, the largest absorption corresponds to the transitions from the IB to the $\mathrm{CB}$. From the electronic structure analysis, these sub-gap transitions are mainly due to transitions between the $\mathrm{X}$ anion orbitals that contribute to both bands. This behavior is different to the general tendency in binary compounds, where the largest transition is usually between the VB and IB because the two band edges have an anion character, whereas the $\mathrm{CB}$ edge has a cation character.

\section{Conclusions}

The substitution of group IV elements by $\mathrm{Ga}$ or $\mathrm{Cu}$ in the chalcopyrites $\mathrm{GaCuX}_{2}(\mathrm{X}=\mathrm{S}$ and $\mathrm{Se}$ ) leads to the formation of a partially full IB in almost all cases. Generally this band is mainly made up of anion orbitals, with a small contribution from the orbitals of the impurity atoms. Consequently, the optical properties of the chalcopyrite are affected slightly: two new sub-gap absorptions, from the VB to the IB and from the IB to the $C B$, appear in addition to the usual one from the $\mathrm{VB}$ to the $\mathrm{CB}$. They would increase the theoretical radiative limiting efficiencies with respect to those of the host semiconductor.

\section{Acknowledgments}

This work has been supported by the GENESIS FV project of the National Spanish program CONSOLIDER (CSD2006-0004), by the European Commission through the funding of the project IBPOWER (Ref. N: Grant Agreement 211640), and by La Comunidad de Madrid through the funding of the project NUMANCIA-2 (Ref. N: S-2009/ ENE-1477). The author is grateful to D. Fuertes and A. Martí for helpful discussions.

\section{References}

[1] K. Zweibel, Prog. Photovoltaics 3 (1995) 279

[2] F. Karg, H. Aulich, W. Riedl, Proceedings 14th European Photovoltaic Solar Energy Conference, Barcelona, Spain, 1997, p. 2012.

[3] B. Dimmler, H.W. Schock, Prog. Photovoltaics 4 (1996) 425.

[4] D. Guimard, N. Bodereau, J. Kurdi, J.F. Guillemoles, D. Lincot, P.P. Grand, M. BenFarrah, S. Taunier, O. Kerrec, P. Mogensen, Proceedings of the 3 rd World Conference on Photovoltaic Energy Conversion, Osaka, Japan, 1, 2003, p. 515.

[5] J.M. Raulot, C. Domain, J. Guillemoles, Phys. Rev. B 71 (2005) 035203.

[6] D. Fuertes Marrón, A. Martí, C. Tablero, E. Antolín, E. Cánovas, P. García-Linares, A. Luque, Proceeding of the 23th European Photovoltaic Solar Energy Conference, Valencia, Spain, 2008, p. 33.

[7] D. Fuertes-Marrón, A. Martí, A. Luque, Thín Solid Films 517 (2009) 2452.

[8] D. Fuertes-Marrón, A. Martí, A. Luque, Phys. Status Solidi A 206 (2009) 1021.

[9] C. Tablero, D. Fuertes Marrón. J. Phys. Chem. C 114 (2010) 2756.

[10] C. Tablero, Chem. Phys. Lett. (2010), doi:10.1016/j.cplett.2010.09.018.

[11] A. Luque, A. Marti, Phys. Rev. Lett. 78 (1997) 5014.

[12] A. Luque, A. Martí, Prog. Photovoltaics 9 (2001) 73.

[13] W. Shockley, H.J. Queisser, J. Appl. Phys. 32 (1961) 510.

[14] Luque, A. Marti, C. Stanley, N. Lopez, L. Cuadra, D. Zhou, J.L. Pearson, A. McKee, J. Appl. Phys. 96 (2004) 903.

[15] K.M. Yu, W. Walukiewicz, J.W. Ager III, D. Bour, R. Farshchi, O.D. Dubon, S.X. Li, I.D. Sharp, E.E. Haller, Appl. Phys. Lett. 88 (2006) 092110.

[16] W. Wang, A.S. Lin, J.D. Phillips, Appl. Phys. Lett. 95 (2009) 011103.

[17] S. Suraprapapich, S. Thainoi, S. Kanjanachuchai, S. Panyakeow, Sol. Energy Mater. Sol. Cells 90 (2006) 2968. 
[18] A. Martí, D. Fuertes Marrón, A. Luque, J. Appl. Phys, 103 (2008) 073706.

[19] C. Tablero, J. Appl. Phys. 106 (2009) 073718.

[20] R.M. Martin, Electronic Structure, Cambridge University Press, Cambridge University Press, 2004.

[21] K. Ohno, K. Esfarjani, Y. Kawazoe, Computational Materials Science, From ab initio to Monte Carlo Methods, Springer, 1999.

[22] R.G. Parr, W. Yang, Density functional theory of atoms and molecules, Oxford, New York, 1989.

[23] J.F. Dobson, G. Vignale, M.P. Das, Electron density functional theory, recent progress and new directions, Plenum Press, New York, 1998.

[24] P. Hohenberg, W. Kohn, Phys. Rev. B 136 (1964) 864

[25] W. Kohn, LJ. Sham, Phys. Rev. 140 (1965) A1133.
[26] J.M. Soler, E. Artacho, J.D. Gale, A. García, J. Junquera, P. Ordejon, D. Sánchez-Portal, J. Phys. Condens. Matter 14 (2002) 2745 and references therein.

[27] D.M. Ceperley, B.]. Alder, Phys. Rev. Lett. 45 (1980) 566.

[28] N. Troullier, J.L. Martins, Phys. Rev. B 43 (1991) 1993.

[29] L. Kleinman, D.M. Bylander, Phy. Rev. Lett. 48 (1982) 1425.

[30] D.M. Bylander, L. Kleinman, Phys. Rev. B 41 (1990) 907.

[31] O.F. Sankey, D.J. Niklewski, Phys. Rev. B 40 (1989) 3979.

[32] J.E. Jaffe, Alex Zunger, Phys. Rev. B 28 (1983) 5822.

[33] S. Chen, X.G. Gong, S.-H. Wei, Phys. Rev. B 75 (2007) 205209.

[34] S. Laksari, A. Chahed, N. Abbouni, O. Benhelal, B. Abbar, Comput. Mater. Sci. 38 (2006) 223. 